\title{
Pseudomonas Oryzihabitans bacteremia in a child with sickle cell disease
}

\author{
Ali M Abu Taleb ${ }^{1}$, Omar E Masmali ${ }^{1}$, Adeeb A Ageel ${ }^{2 *}$ and Haider M Arishi ${ }^{3}$ \\ ${ }^{1}$ Department of pediatrics, King Fahad Hospital, Saudi Arabia \\ ${ }^{2}$ Division of hematology, King Fahad Hospital, Saudi Arabia \\ ${ }^{3}$ Division of infectious diseases, King Fahad Hospital, Saudi Arabia
}

\section{Case report}

11-years old boy. At age of one year, he was diagnosed as case of Sickle Cell Disease (SCD). At age of 10 years, he was diagnosed to have avascular necrosis of the right hip. He was admitted to our institution with history of pain of right hip which started five days prior to admission. This pain was gradual in onset, moderate in severity with limitation of movements of the hip joint.

The pain increased with weight bearing and decreased with rest. It was not radiating elsewhere or related to specific time. There was no affection of other joints. There was no history of fever, skin rash or change urine color. There was neither history of trauma, animal contact nor raw milk ingestion. He was admitted as case of sickle cell anemia with vaso-occlusive crisis.

After one day of hospitalization, the pain improved on the right hip joint and started in the left hip side which radiated to the left knee with limitation of movement. Past history revealed twice blood transfusions; the last one was 3 months before the current admission. Physical examination revealed a child who was in pain, score of 8 (Wong Baker pain scale, range $0-10$ with 10 , it hurts and worst), conscious, alert, with no pallor or jaundice. The chest and heart examination was within normal. There was no organomegaly. His body weight was 26 kilograms, above the $10^{\text {th }}$ percentile; his height 130 centimeters on the $10^{\text {th }}$ percentile; his temperature $36.7^{\circ} \mathrm{C}$.

The joint examination showed tenderness on the left hip area along the anterior surface of left thigh involving the knee with decrease range of motion, no swelling, erythema or hotness compared with other side. There was no joint or limb deformities were noted. Laboratory studies revealed leukocytosis, WBC count $15.9 \times 10^{9} / \mathrm{L}$ (neutrophils $53.9 \%$ lymphocytes $31.9 \%$ monocytes $10.8 \%$ eosinophils $2.7 \%$ ), hemoglobin (Hb) $80 \mathrm{~g} / \mathrm{L}$ and platelets $429 \times 10^{9} / \mathrm{L}$. Erythrocyte Sedimentation Rate (ESR) $17 \mathrm{~mm} / \mathrm{h}$ (normal $<30 \mathrm{~mm} / \mathrm{h}$ ) and C-Reactive Protein (CRP) $0.5 \mathrm{mg} / \mathrm{dL}$ (normal $<0.5 \mathrm{mg} / \mathrm{d}$ ). Blood culture grew Pseudomonas oryzihabitans, which was resistant to Ceftazidime and sensitive to amikacin, aztreonam, cefepime, gentamicin, imipenem, eropenem, piperacillin-tazobactam and trimethoprim-sulfamethoxazole. Urine culture was negative. Hemoglobin electrophoresis: $\mathrm{Hb}$ A :00, $\mathrm{HbF}$ 15, Hb S 82 and Hb A2 3\% respectively. Reticulocytes $10.03 \%$. HIV screening, HBsAg and $\mathrm{HCV}$ anti-bodies were negative. Magnetic Resonant Imaging (MRI) with contrast of left hip joint demonstrated a moderate degree of altered marrow signal intensity changes involving the femoral head epiphysis with mild joint effusion consistent with acute avascular necrosis and reactive synovitis. There was a similar finding involving the right hip in keeping with avascular necrosis. The patient was given analgesics including acetaminophen, ibuprofen and intravenous morphine regularly. He continued developing severe pain which ultimately required blood transfusions, initially simple and then partial blood exchange. For Pseudomonas Oryzihabitans bacteremia, the child was treated by intravenous piperacillin-tazobactam for 7 days. The repeated blood culture was negative after 48 hours. After treatment of pain, he was discharged home in stable condition. On follow up two, four and eight weeks after discharge, the child was in his usual steady state, afebrile with mild residual pain on mobilization.

\section{Discussion}

Pseudomonas oryzihabitans is the current name for the bacteria that was called Chromobacterium typhiflavum and Flavimonas oryzihabitans [1].

It's gram negative, oxidase negative, non-lactose fermenting rodshaped bacteria which produces yellow pigmented colonies on agar media. It survives in moist environment including rice paddies [2]. The source for the infection is probably environmental [3]. P. oryzibanitans has been associated with several outbreaks in hospitals and considered as a nosocomial agent $[4,5]$. The organism was isolated from hospital sinks, inhalational therapy equipment and saline gauze canister $[2,4]$. It has caused central venous catheter infection as well [6].

In 1977, The first case of bacteremia caused by this organism was reported in a patient who underwent surgical evacuation of extradural cerebral hemorrhage due to sever compound skull fracture [7]. The organism can cause bacteremia, CNS infection, peritonitis, pneumonia, UTI, hip infection, abscesses, wounds and soft tissue infections [3,7-10].

In pediatric age group, infections due $P$. oryzihabitans were reported in patients with leukemia, lymphoma, neuroblastoma, brain tumors, aplastic anemia, kwashiorkor, congenital heart disease and Hirschsprung's disease [11]. In patients with SCD, the $1^{\text {st }}$ case of $P$. oryzihabitans bacteremia was reported in 1991 for 37 years old woman who was admitted with the acute onset sickle cell crisis complicated by multiple central venous catheter related infections [12].

${ }^{\star}$ Correspondence to: Haider M Arishi, Head, Pediatric Infectious Diseases, Department of Pediatrics, King Fahad Central Hospital, P O BOX 204, Jazan 1991, Saudi Arabia, E-mail: drarishih@gmail.com

Key words: pseudomonas, oryzihabitans, sickle cell, bacteremia, child

Received: October 01, 2020; Accepted: October 06, 2020; Published: October 09,2020 
To the best of our knowledge, this is the first case of Pseudomonas oryzihabitans bacteremia in a child with SCD. Our patient was treated with a short course of pipracillin-tazobactum. Follow up up to 8 weeks, he did not develop any fever or clinical evidence of bone infection. The patient was referred to specialized center for potential bone marrow transplant.

\section{References}

1. Nei T, Sonobe K, Onodera A, Itabashi T, Yamaguchi H, et al. (2015) Two cases with bacteremia suspected to be due to relatively rare Pseudomonas (Flavimonas) oryzihabitans. J Infect Chemother 21: 751-755.

2. Kentaro K, Norio K, Komagata K (1985) Two new species of Pseudomonas: $P$ oryzihabitans isolated from rice paddy and clinical specimens and $P$. luteola isolated from clinical specimens. Int J Syst Bacteriol 35: 467-474.

3. Tena D, Fernández C (2015) Pseudomonas oryzihabitans: an unusual cause of skin and soft tissue infection. Infect Dis 47: 820- 824.

4. Woo KS, Choi JL, Kim BR, Kim JE, Kim KH, et al. (2014) Outbreak of Pseudomonas oryzihabitans pseudobacteremia related to contaminated equipment in an emergency room of a tertiary hospital in Korea. J Infect Chemother 46: 42-44.
5. Eom JS, Cheong HJ, Kim WJ (2009) Pseudobacteremia outbreak of Pseudomonas oryzihabitans in an emergency department of a tertiary hospital in Korea. Infect Control Hosp Epidemiol 30: 803-804.

6. Marín M, Garca de Viedma D, Martn-Rabadn P, Rodrguez-Crixems M, Bouza E (2000) Infection of hickman catheter by Pseudomonas (formerly Flavimonas) oryzihabitans traced to a synthetic bath sponge. J Clin Microbiol 38: 4577-4579.

7. Pien FD (1977) Group VE-2 (Chromobacterium typhiflavum) bacteremia. J Clin Microbiol 6: 435-436.

8. Bhatawadekar SM (2013) Community-acquired urinary tract infection? by Pseudomonas Oryzihabitans. J Glob Infect Dis 5: 82-84.

9. Panagopoulos GN, Megaloikonomos PD, Liontos M, Giannitsioti E, DrogariApiranthitou M, et al. (2016) Pseudomonas oryzihabitans infected total hip arthroplasty. J Bone Jt Infect 1: 54-58.

10. Giacometti A, Cirioni O, Quarta M, Schimizzi AM, Del Prete MS, et al. (1998) Unusual clinical presentation of infection due to Flavimonas oryzihabitans. Eur J Clin Microbiol Infect Dis 17: 645-648.

11. Reed RP (1996) Flavimonas oryzihabitans Sepsis in Children. Clin Infect Dis 22: 733-734

12. Decker CF, Simon GL, Keiser JF (1991) Flavimonas oryzihabitans (Pseudomonas oryzihabitans; $\mathrm{CDC}$ group Ve-2) bacteremia in the immunocompromised host. Arch Intern Med 151: 603-604.

Copyright: $\odot 2020$ Ali M Abu T. This is an open-access article distributed under the terms of the Creative Commons Attribution License, which permits unrestricted use, distribution, and reproduction in any medium, provided the original author and source are credited. 